\title{
Intracellular Signaling Pathways Involved in Cell Differentiation in Trypanosomatids
}

\author{
Angela H. Lopes* ${ }^{*}$, , Marta T. Gomes ${ }^{2}$, Fernando L. Dutra ${ }^{1}$, Alane B. Vermelho ${ }^{1}$, \\ José R. Meyer-Fernandes ${ }^{2}$, Mário A. C. Silva-Neto ${ }^{2}$, Thaïs Souto-Padrón ${ }^{1}$ and Danielle P. Vieira ${ }^{1}$ \\ ${ }^{I}$ Instituto de Microbiologia Prof. Paulo de Góes, UFRJ; Cidade Universitária, Ilha do Fundão, Rio de Janeiro, R.J. \\ 21941-590, Brazil \\ ${ }^{2}$ Instituto de Bioquímica Médica, UFRJ; Cidade Universitária, Ilha do Fundão, Rio de Janeiro, R.J. 21941-590, Brazil
}

\begin{abstract}
Knowledge of cell signaling pathways in trypanosomatids is crucial for the future design of new drugs to treat diseases caused by these parasites. The publication of the complete genome sequences of three pathogenic trypanosomatids, Trypanosoma brucei, T. cruzi and Leishmania major, revealed numerous protein members of signaling pathways that modulate important processes, such as cell differentiation. Even so, little is known about the role that these proteins play in the physiology of trypanosomatids. This review aims to stimulate discussion on this subject to encourage further studies of the signaling pathways involved in the cell differentiation of trypanosomatids.
\end{abstract}

Keywords: Cellular differentiation, signaling pathways, protein kinases, Trypanosoma cruzi, Trypanosoma brucei, Leishmania.

\section{INTRODUCTION}

Cell differentiation during the life cycle of trypanosomatid parasites comprises integrated changes in cell morphology, metabolism, gene expression and signaling pathways. These processes are different from most other eukaryotic life forms, especially the multicellular organisms. While in higher eukaryotes cell differentiation is a "one way" phenomenon, in trypanosomatids the characteristic morphotypes of a given genus are interchangeable throughout its life cycle, adjusting to environmental factors present in each one of their hosts [1]. These structural and physiological changes are progressive and result in altered infectivity, a critical feature of a parasite [2]. The morphotypes that characterize a certain genus are cell shape, dimensions and the position of the complex kinetoplast-flagellar pocket relative to the nucleus [3,4] (Fig. 1).

The complex morphological and biochemical changes during cell differentiation in trypanosomatids are directed by different ligands and/or stimulatory molecules present in their environment $[5,6]$. These changes are mediated by signaling pathways that coordinate processes such as cell growth, development and differentiation. In mammalian systems, this coordination involves several signaling scaffolds, such as protein kinases and phosphatases, G proteins and second messengers. Interestingly, some receptors for mammalian ligands have been described in a few trypanosomatids [5], although genes encoding for $G$ proteins have not been found in their genomes [7-9]. The presence of receptors for several vertebrate extracellular signaling molecules has been well documented $[5,6]$. These signaling molecules display a

*Address correspondence to this author at the Instituto de Microbiologia Prof. Paulo de Góes, UFRJ; Cidade Universitária, Ilha do Fundão, Rio de Janeiro, R.J. 21941-590, Brazil; Tel: + 55-21-2562-6740;

Fax: + 55-21-2560-8344; E-mail: angela.lopes@ micro.ufrj.br wide range of activities, such as the induction or inhibition of target cell survival, proliferation and differentiation. When directly interacting with different parasites, these molecules exert similar activities as they do in mammals [6].

Protozoa in the genus Herpetomonas are typically taxonomically characterized by the presence of promastigote, paramastigote and opisthomastigote developmental stages [10] (Fig. 1). This genus is characterized by a life cycle involving only an invertebrate host, although in some cases these protozoans can parasitize plants; some Herpetomonas species can be human pathogens, causing a diffuse cutaneous leishmaniasis-like infection in immunodeficient and immunocompetent patients $[11,12]$. Platelet-activating factor (PAF), a naturally-occurring phospholipid mediator [13], triggers the cell differentiation of $H$. muscarum muscarum and $T$. cruzi, which is completely abrogated by a specific PAF receptor antagonist (WEB 2086), indicating that these parasites may have receptors for PAF $[14,15]$. Additionally, PAF-stimulation of cell differentiation in $H$. m. muscarum involves a complex signal transduction pathway that includes Ser/Thr and Tyr protein phosphatases, PLC, PKC and MAP kinases as upstream elements as well as CK2 as a target. This study demonstrated for the first time that a lipid mediator could stimulate the activation of $\mathrm{CK} 2$, leading to parasite differentiation [16]. The isolation of a PAF-like synthesized by $T$. cruzi, which stimulates the differentiation of the parasite itself from epimastigotes to metacyclic trypomastigotes, highly infective to mammalian cells [17], suggests that PAF-triggered cell differentiation in trypanosomatids is a phenomenon that may actually occur in nature.

Genomic and proteomic studies of T. brucei, T. cruzi and $L$. major have allowed for a description of the entire kinome for these trypanosomatids [18]. This analysis demonstrated that although these protozoans lack members of the receptorlinked or cytosolic tyrosine kinase families, they have abun- 
dant quantities of the STE and CMGC families of protein kinases [18]. The STE and CMGC families of protein kinases are involved in regulating cell cycle control, responses to stress during their complex life cycles and cell differentiation $[7-9,18]$.

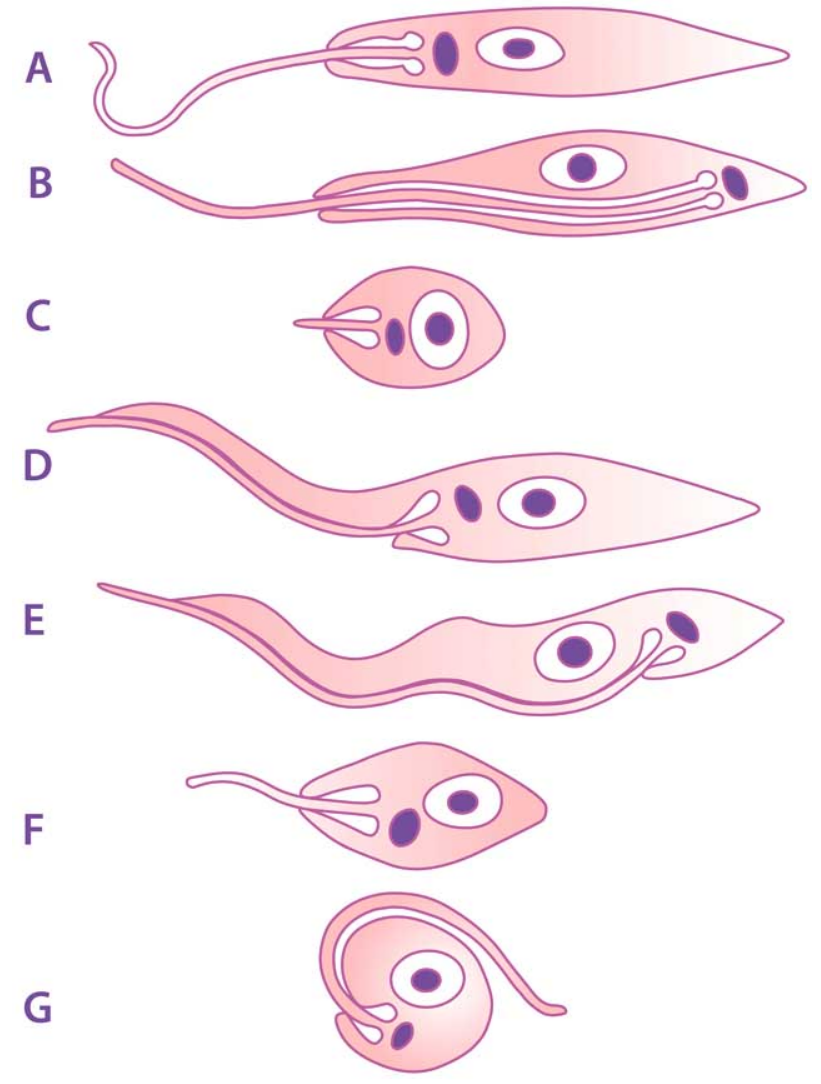

Fig. (1). The most common morphotypes of trypanosomatids. A: promastigote; B: opisthomastigote; C: amastigote; D: epimastigote; E: trypomastigote; F: choanomastigote; G: spheromastigote. Diagram based on Hoare and Wallace [3].

\section{Trypanosoma cruzi}

Trypanosoma cruzi, the etiological agent of Chagas disease, has a complex life cycle, alternating between a triatomine insect vector and a mammalian host. Epimastigote forms, after replicating in the insect midgut, differentiate into infective metacyclic trypomastigotes, which are released with feces when the insect feeds on the mammalian host, thus enabling infection of the latter and completion of the parasite's life cycle [19]. The differentiation enables $T$. cruzi to adapt to environmental changes through morphological (Fig. 2) and functional transformations that involve the activation of protein kinase cascades and a variety of complex physiological signals [6]. Little is known about the potential of the mammalian or vector-derived molecules to regulate the growth and differentiation of the parasites. Even so, a number of in vitro systems have been proposed to induce metacyclogenesis, including the use of artificial triatomine urine media [20].

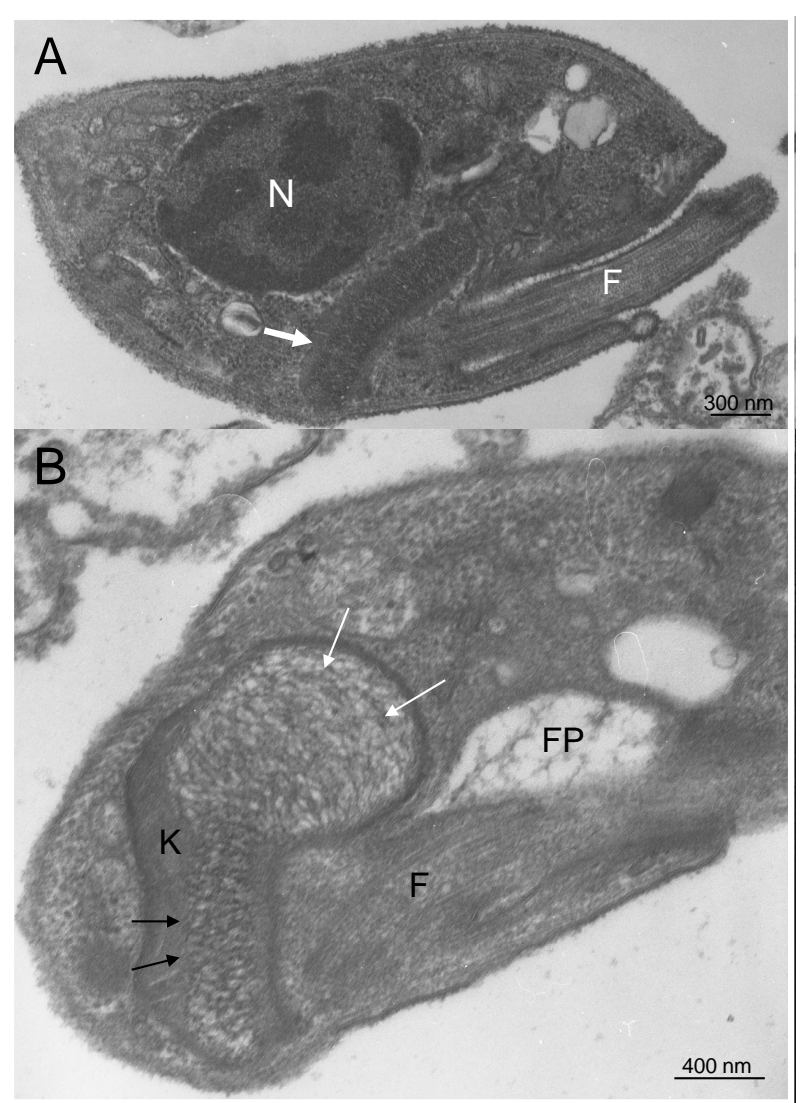

Fig. (2). Cell differentiation of Trypanosoma cruzi. A. Transitional form between amastigote and trypomastigote obtained from the supernatant of LLC-MK2 cell culture previously infected with trypomastigotes. The kinetoplast (white arrow) although showing a morphology (bar- or rod-shaped) typical of the amastigote form, is located close to the nucleus. $\mathrm{N}=$ nucleus; $\mathrm{F}=$ flagellum. $\mathbf{B}$. Transitional form between epimastigote and trypomastigote obtained from a stationary growth phase culture. The kinetoplast $(\mathrm{K})$ shows a morphology in which part of the DNA fibers are arranged as in epimastigotes (black arrows) and part as in trypomastigotes (dispersed filaments - white arrows). $\mathrm{FP}=$ flagellar pocket; $\mathrm{F}=$ flagellum. T. cruzi epimastigotes of the CL-Brener clone were maintained at $28^{\circ} \mathrm{C}$ by weekly transfers in liver infusion tryptose (LIT) medium supplemented with $10 \%$ fetal calf serum (FCS). Four-day-old culture forms at the mid-log phase of growth were used in all experiments. Tissue culture trypomastigotes and amastigotes were obtained from the supernatants of 5 to 6-day-old infected LLC-MK2 cells maintained in RPMI-1640 medium supplemented with $2 \%$ FCS at $37^{\circ} \mathrm{C}$ in a $5 \%$ humidified $\mathrm{CO}_{2}$ atmosphere [21]. The parasites in $\mathbf{A}$ and $\mathbf{B}$ were prepared for transmission electron microscopy as described [21]. Ultra-thin sections obtained with a Leica (Nussloch, Germany) ultramicrotome were stained with uranyl acetate and lead citrate, and observed using a FEI Morgagni F 268 (Eindhoven, The Netherlands) transmission electron microscope operating at $80 \mathrm{kV}$ [21].

Fraidenraich and co-workers [22] showed that intestinal extracts of Triatoma infestans, containing peptides derived from hemoglobin breakdown, stimulated adenylate cyclase and induced metacyclogenesis. In addition, peptides released from the proteolysis of fibronectin increased cAMP levels in 
trypomastigotes [23]. cAMP analogs and phosphodiesterase inhibitors have also been reported to induce the differentiation of this parasite [24,25] because proliferation decreased due to decreased DNA or RNA synthesis [26,27]. In trypanosomatids, like in most eukaryotes, cAMP is synthesized by adenylyl cyclases (ACs) and degraded by phosphodiesterases (PDEs) [28]. In most mammalian systems, cAMP regulates numerous physiological processes through cAMPdependent protein kinase (PKA). By binding the regulatory subunit of the enzyme and releasing it from the catalytic subunit, cAMP stimulates PKA activity; however, little is known about PKA in trypanosomatids. A putative regulatory subunit of PKA was identified in $T$. bruce $i$ with an associated kinase activity [29], but cGMP and not cAMP stimulated this kinase activity [29]. The presence of PKA activity was also suggested in T. cruzi [30] and the expression of this putative PKA catalytic subunit appears to be developmentally regulated [31]. Putative PKA catalytic subunits have also been identified in $L$. major and $L$. donovani $[28,32,33]$. T. cruzi genome contains all of the members of the cAMP cascade, such as adenylate cyclase, phosphodiesterases and catalytic and regulatory subunits of PKA [7-9,34]. Additionally, trypanosomatid adenylyl cyclases have a completely different structure in comparison to their mammalian counterparts. T. cruzi adenylyl cyclase is similar in structure to receptor guanylate cyclases and contains a putative extracellular ligand-binding domain and a cytoplasmic domain. The extracellular domain of the receptor may directly activate the intracellular components of the adenylyl cyclase without requiring activation by heterotrimeric G-proteins or other regulatory factors $[35,36]$. Even though $\mathrm{G}$ proteins cannot be found in the now complete trypanosome genomes [7-9], Coso and co-workers [37] characterized a Gi-protein from $T$. cruzi epimastigote membranes. Blocking the PKA function using a genetic approach allowed them to identify some putative proteins that interact with the PKA catalytic subunit of T. cruzi (TcPKA), such as PI3 kinase, a mitogen-activated extracellular signal-regulated kinase (MAPK) and a cAMPspecific phosphodiesterase (PDEC2) [28,37]. Intriguingly, an adenylyl cyclase stimulated by calcium, which interacts with paraflagellar rod protein [38] was suggested to be one of these proteins.

"Crosstalk" between adenylyl cyclase and phospholipase $\mathrm{C}$ during events of growth and differentiation in $T$. cruzi has also been described. T. cruzi exposure to mitogenic factors in fetal calf serum (FCS) promoted the inhibition of cAMP production and stimulated phospholipase C (PLC), leading to the accumulation of inositol phosphates (InsP) and diacylglycerol (DAG) [27]. In a similar way, chicken $\alpha^{\mathrm{D}}$-globin was shown to produce dose-dependent inositol triphosphate (InsP3) accumulation as a consequence of phosphatidylinositol phosphate-PLC (PtdIns-PLC) activation [39] and transient calcium mobilization [40]. Lipids and lipid kinases related to the inositol cycle were shown to display important roles in signal transduction processes, such as cell differentiation [40]. Wainszelbaum and co-workers [41] showed that free fatty acids (FFA), primarily oleic acid (OA), found in the intestinal tract of $T$. infestans induced $T$. cruzi metacyclogenesis through a signaling pathway involving de novo diacylglycerol biosynthesis, protein kinase $\mathrm{C}$ (PKC) activation and $\mathrm{Ca}^{2+}$ mobilization [41]. The PKA pathway does not appear to be activated in these cells, as previously described by Fraidenraich and co-workers [22]. It is possible that activation of the PKA pathway is blocked in the presence of the PKC-activating stimuli. Thus, activation of one signaling pathway may block an alternative pathway, leading to the same differentiating effects [41]. In addition, these authors found that OA directly stimulated the in vitro activity of $T$. cruzi PKC in a dose-dependent manner and induced the selective translocation of PKC isoenzymes. Interestingly, PKC isoforms were differentially expressed in the infective and non-infective $T$. cruzi stages, suggesting that each isoenzyme possesses specific functions that could be related to the diverse environments that the parasite encounters during its complex life cycle [42]. Indeed, activation of stage-specific genes during metacyclogenesis was documented [43]. Nevertheless, very little is known about the differentiation process and the changes that occur during the stage-regulated gene expression program.

Platelet-activating factor (PAF) was shown to promote the enhancement of the secreted phosphatase activity through the activation of PKC in T. cruzi [44] and a PAF-like phospholipid produced by these parasites triggered its own cell metacyclogenesis [17]. The signaling pathways are modulated through the antagonistic activities of highly specific protein kinases and protein phosphatases that control a number of processes, including metabolic pathways, cell-cell communication, cell growth and proliferation and gene transcription. Analyses of T. cruzi phosphatome identified 86 phosphatase catalytic domains in these parasites [45].

Recently, Nakayasu et al. [46] sequenced 221 phosphorylation sites in 119 proteins from T. cruzi. Of those 221 newly identified phosphorylation sites, $148(65.5 \%)$ were on serines, $57(25.2 \%)$ on threonines and $8(3.5 \%)$ on tyrosines. The identified phosphoproteins were categorized based on their role in cell structure, motility, transportation, metabolism, pathogenesis, DNA/RNA/protein turnover and signaling [46]. In addition, approximately $2 \%$ of the $T$. cruzi genome encodes protein kinases, suggesting a major regulatory role in controlling parasite development and function [47].

\section{Trypanosoma brucei}

Trypanosoma brucei is a protozoan flagellate that causes sleeping sickness in tropical Africa and is responsible for $\sim 30,000$ deaths per year [19]. The life cycle of T. brucei alternates between the midgut of the tsetse fly vector and the bloodstream of mammalian hosts. These trypanosomes are pleomorphic in the mammalian bloodstream, transforming from a replicating long slender form into a non-dividing short stumpy form pre-adapted for transmission into the tsetse fly. The differentiation is signaled through a densitysensing mechanism as parasite numbers increase in response to an unidentified parasite derived signaling factor, stumpy induction factor (SIF) [48,49]. In the midgut of the vector, the short stumpy form differentiates into a replicating procyclic form, migrating to the salivary glands and differentiating into infective forms [50]. The most remarkable marker for differentiation of these parasites is the exchange of the main surface antigens: variant surface glycoprotein (VSG) in the bloodstream stage [51] and procyclin in the procyclic stage [52]. Both proteins are attached to the cell surface by a glycosylphosphatidylinositol (GPI)-anchor [53]. VSG is a 
homodimer that surrounds the cell and forms a surface coat that is impenetrable to host serum macromolecules. Procyclin genes encode a more restricted family composed of two basic isoforms, EP and GPEET, defined by amino acid sequences of C-terminal repeat domains, glu-pro for EP and gly-pro-glu-glu-thr for GPEET [52,54,55]. These domains confer resistance to hydrolysis while the parasite is located in the vector. In vitro experiments showed that 12 hours after the initial stimulus for differentiation, the surface coat is complete and differentiating trypanosomes enter into their first cell cycle as fully transformed procyclics [56]. During the differentiation process, the VSG coat is actively removed through GPI hydrolysis and endoproteolysis [57-59]. GPI hydrolysis is mediated by an endogenous GPI-specific phospholipase C (GPI-PLC) that is found exclusively in the bloodstream stage of the parasite $[60,61]$. The VSG GPIanchor is present early in the differentiation process in the starting stumpy population. The other mode of release, endoproteolysis, is mediated by a zinc metalloprotease that is up regulated during differentiation [59]. A zinc metalloprotease gene (MSP), coding for three different proteins (MSP$\mathrm{A},-\mathrm{B},-\mathrm{C})$ that are differentially expressed into the differentiation forms, was found in the T. brucei genome [62,63]. In addition, to prevent loss of the newly synthesized procyclin coat, the structure of the GPI anchor precursor that is attached to surface proteins must change. The structure changes from that found in the bloodstream cells, which is susceptible to GPI-PLC activity $[64,65]$, to that found in procyclic parasites, which is resistant [66]. There are differences in GPI anchors in the different forms and the bloodstream form has a diacylglycerol structure, while the procyclic form presents a lysoacylglycerol acylinositol structure. Thus, the GPI biosynthetic shift prevents the newly synthesized procyclin from being shed [56]. Many factors have been shown to induce the differentiation process in vitro; for example, the addition of the Krebs cycle intermediate citrate/cis-aconitate (CCA) to the culture medium speeds up the rate of differentiation [67-69]. A variation in the temperature range from $37^{\circ} \mathrm{C}$ to $20^{\circ} \mathrm{C}$ induces the immediate and reversible expression of the insect stage-specific EP procyclin surface protein in slender and stumpy-stage bloodstream trypanosomes via a post-transcriptional mechanism. In stumpy cells, the cold shock elicits up to a 1000 -fold increased sensitivity to CCA [70]. To differentiate into the next developmental stage, parasites must have evolved mechanisms to sense and respond to the microenvironment imposed by their different hosts to adapt their biology to extracellular or intracellular survival [71]. These mechanisms are posttranslational events like signaling pathways that are mediated by proteins kinases and phosphatases. A recent study of the phosphoproteome of $T$. brucei showed that most $T$. brucei phosphorylation sites were detected on serine residues (75\%) and threonine residues (21.5\%) and, to a lesser extent, on tyrosine residues (3.5\%). Of the $491 \mathrm{~T}$. brucei phosphoproteins viewed in the phosphoproteome study, 44 were kinases [72]. The recent kinome study showed the existence of 170 ePKs (conventional protein kinases) and 12 aPKs (atypical protein kinases) [72]. The trypanosomatid kinome lacks members of the receptor-linked or cytosolic tyrosine kinase families, but there is evidence that several proteins are phosphorylated on tyrosine residues in kinetoplastids [73, 74], presumably though the activity of dual-specificity pro- tein kinases [such as Wee and dual specificity tyrosine phosphorylation-regulated kinase (DYRK) family kinases] $[47,72]$. Tyrosine phosphatase activity also shows marked differences among different life cycles stages in T. brucei [75]. The trypanosomatid-specific protein tyrosine phosphatase TbPTP1 has a prominent role in the differentiation process. When TbPTP1 activity is inhibited by RNAi or biochemically inhibited with the specific inhibitor BZ3, the process of differentiating into procyclic forms occurs spontaneously in the absence of any exogenous trigger. Thus, the form shows all of the markers from the procyclic stage, such as EP and GPEET procyclins and the stage-specific cytoskeletal antigen CAP5.5 [76]. The stumpy cells were uniformly arrested in G1/G0 and initiated differentiation synchronously and efficiently [77]. The enzyme $T b P T P 2$ seems not to contribute to the observed differentiation phenotype because RNAi against this enzyme did not elicit differentiation of the bloodstream forms [76], which suggests that TbPTP1 normally inhibits this differentiation step [76,78]. Although only a small proportion of cultured monomorphic bloodstream forms were differentiated, uniformly arrested stumpy forms differentiated with an efficiency of nearly $100 \%$ when exposed to the inhibitor. This result suggested a model in which cell-cycle arrest was required for differentiation and in which this arrest was prevented by the activity of $T b$ PTP1 in the bloodstream. Once in the fly, inactivation of this enzyme would allow those cells that were able to differentiate to do so [2]. The trypanosomatid kinome has an abundance of STE and CMGC family protein kinases, which include the members of the canonical MAP kinase cascade (MAPKs, MAPKKs and MAPKKKs) involved in environmentally regulated cell cycle control, differentiation and the cellular response to various stress signals [18]. These two families alone make up more than $40 \%$ of the conserved trypanosomatid kinome [76]. Various members of the MAPK family and MAPK-like kinases were found in T. brucei. The first MAPK to be identified and characterized, KFR1, is essential for procyclic forms. The bloodstream form of KFR1 shows an increased abundance of mRNA and proteins compared with the procyclic form [79]. Gene-targeting experiments revealed that MAPKs were directly implicated in the growth control and differentiation of $T$. brucei. Null mutants of TbMAPK2, the ortholog of MPK 4 in Leishmania, proliferated normally in cultures in the bloodstream stage but differentiated less efficiently in the procyclic insect stage, resulting in non-synchronous cell cycle arrest [80]. Genetic deletion of TbMAPK5, the ortholog of MPK 5 of Leishmania, did not interfere with the proliferation of $T$. brucei at the procyclic stage, both in cultures and during the development of the tsetse fly [81]. However, the absence of TbMAPK5 resulted in low-rate infections in mice, accompanied by premature differentiation in the non-proliferative stumpy form. These results suggest that TbMAPK2 and TbMAPK5 may have complementary roles in the control of proliferation in procyclic and bloodstream T. brucei developmental stages, respectively. The cyclin-dependent kinase (CDK) family is comparatively large in trypanosomatids, with 11 members in T. brucei. CDK requires an activating cyclin partner for activity and analysis of the genome revealed 10 orthologous cyclins in the parasite (CYC2-CYC11) [43]. Some CDKs require phosphorylation in the conserved threonine residue by a cdc2-activating kinase (CAK). Down-regulation of 
CRK3 (cdc2-related kinase protein) in both the procyclic and the bloodstream form resulted in a mitotic block and growth arrest [82]. It is currently postulated that cAMP plays an important role in the differentiation from slender to stumpy forms, although much of the data to support this hypothesis is circumstantial. Some genes within the T. brucei kinome have been found to be related to two genes in the AGC family (Tb09.211.2410 and $\mathrm{Tb} 11.02 .2210$ ) that may code for proteins related with the catalytic and regulatory PKA subunits, respectively [72]. In T. brucei, two distinct peaks of adenylyl cyclase activity were observed during differentiation from the bloodstream form to the procyclic form. It has been suggested that the use of an analog of cAMP, 8pCPT-cAMP, induced a slender-to-stumpy differentiation in the pleomorphic bloodstream form [49]. However, a requirement for cAMP or cyclase activity in this or in the subsequent release of variable surface glycoprotein was not apparent $[83,84]$. The use of another cell-permeable analog of cAMP, 8-pCPT-2'- $O$-Me-cAMP, inhibited the proliferation of the bloodstream form, but its PDE hydrolysis-resistant equivalent, $\mathrm{Sp}$-8-pCPT-2'-O-Me-cAMP, does not show this capacity for inhibition. However, the most common downstream product of the hydrolysis of 8-pCPT-2'- $O$-Me-cAMP (8-pCPT-2'-O-Me-5'-AMP/-adenosine) is a more potent inhibitor [85]. High concentrations of extracellular cAMP, 5'-AMP or adenosine did not significantly affect the proliferation of $T$. brucei, suggesting that the antiproliferative effect caused by the nucleotide analogs was mediated by an intracellular "receptor" [85]. Membrane permeable 5'-AMP/ adenosine analogs caused cell-cycle arrest and differentiation of slender forms into stumpy-like forms [85]. These data suggest that the observed effect of cAMP was due to the downstream products of PDE hydrolysis. The T. brucei line that was defective to differentiation (DiD1) showed an increased expression of PAD genes. The codes of the DiD1 were closely related to members of a family of 14 transmembrane-spanning proteins in the major facilitator superfamily [86]. RNAi against these genes was used in a pleomorphic lineage of T. brucei (AnTat 1.1 90:13). When this gene was ablated, the parasite presented a reduced differentiation rate of cells exposed to $\mathrm{CCA}$ and decreased the response of stumpy cells to citrate at $20^{\circ} \mathrm{C}$ [86]. Another $T$. bruce $i$ that had mutated and lacked the TbICP locus encoded for an endogenous cysteine peptidase inhibitor (ICP) and showed marked differences during the differentiation process. The null mutant show an accelerated coat exchange when stimulated with CCA in comparison to the wild type. A synthetic inhibitor of cysteine peptidases (K11777) could act in the same way [87], suggesting a role for the CPs in the differentiation process of the parasite. The proteins TbZFP1 and TbZFP2 that have a RNA-binding zinc finger $\mathrm{CCCH}$ motif could be involved in the differentiation process as well. An RNAi against TbZFP2 inhibited the initiation of the process and overexpression of the RNAi generated a procyclic-specific morphological type [88]. T. brucei showed an alteration in kinetoplast positioning during differentiation; the bloodstream form presented this kinetoplast in the very posterior end, while the procyclic forms presented this kinetoplast half way between the nucleus and the posterior end. Ablation of TbZFP1 caused an alteration in kinetoplast migration in parasites and the null mutant changed the surface markers but maintained the kinetoplast in the same position as the bloodstream form and presented many cell cycle defects [88]. During the differentiating process, an alteration in the intracellular calcium concentration was shown to occur [89]. The functions of the proteins that require the binding of calcium for activity could be regulated in parallel with progression through the cell cycle as a consequence of the alteration in cytosolic calcium levels.

\section{Leishmania}

Leishmania species are the causative agents of leishmaniasis, which currently affects millions of people and threatens 350 million people in 88 countries [90]. Infection in humans may present as different forms of leishmaniasis depending on the Leishmania species causing the infection [91]. The clinical symptoms of this disease range from cutaneous to mucocutaneous to visceral. Some species, such as L. major, L. tropica and L. mexicana, may cause cutaneous forms of the disease that are typically displayed as selfhealing skin ulcers on exposed parts of the body [90]. Infection by species such as L. braziliensis, L. panamensis and $L$. guyanensis may cause mucocutaneous forms of leishmaniasis that initially present as cutaneous lesions but may then spread and lead to partial or total destruction of mucous membranes, which are often resistant to treatment or cure [90]. L. donovani and L. chagasi infections may result in a chronic visceral disease that affects the liver and spleen and are usually fatal if left untreated [90,91].

Leishmania spp. are heteroxenic parasites that present a complex life cycle that includes mammalian and insect hosts; the parasite forms are adapted to the environmental conditions within their hosts $[92,93]$. The promastigote form that resides in the sandfly insect vector occurs in the form of a multiplicative extracellular flagellated cell. The amastigote form resides in macrophages of the mammalian host, to which they are pathogenic. Upon infection of the mammalian host, metacyclic promastigotes are rapidly phagocytized by macrophages and differentiate into the proliferative intracellular amastigote form that survives and multiplies in a parasitophorous vacuole. Within the mammalian host, the amastigote life stage can survive for several months or years $[92,93]$. After transmission into the sandfly vector, the amastigotes rapidly undergo differentiation into the procyclic promastigote form, which colonizes the gut of the sandfly and then migrates to the sandfly foregut. In the foregut, the procyclic promastigote form differentiates into the metacyclic promastigote form and, upon a subsequent blood meal, is transmitted to a mammalian host $[19,91,92,94]$.

Surviving the environmental variations during the life cycle of Leishmania spp. is a challenge that these parasites have overcome, evolving into more adapted and specialized forms. Studies of the differentiation events in Leishmania have mostly examined metacyclogenesis and the differentiation of metacyclic promastigotes into amastigotes in the macrophages of the mammalian hosts. Metacyclogenesis is the differentiation process that turns non-infective procyclic promastigotes into metacyclic infective promastigotes before they are injected by the sandfly vector into the mammalian hosts. Various biochemical changes occur that pre-adapt the parasite to living in the mammalian host. Although the steps for Leishmania differentiation in vitro have been recognized, the signaling pathways and molecular events that mediate 


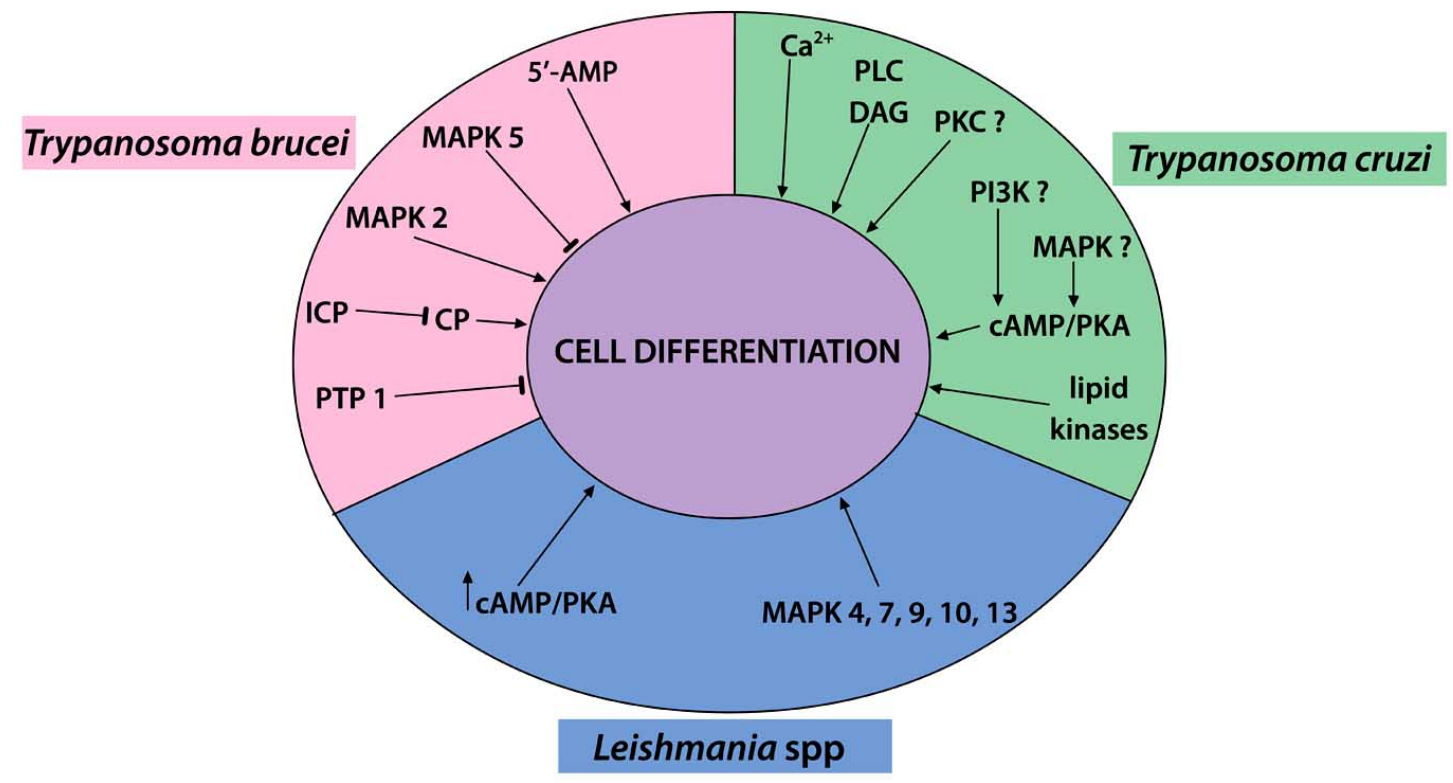

Fig. (3). Intracellular signaling pathways involved with cell differentiation in Leishmania spp., Trypanosoma cruzi and Trypanosoma brucei. The intracellular signaling pathways listed here are summarized in this diagram. ICP - endogenous cysteine peptidase inhibitor; CP cysteine peptidase; PTP - protein tyrosine phosphatase; MAPK- mitogen-activated protein kinase; PLC- phospholipase C; DAG - diacyl glycerol; PKC- protein kinase C; PI3K- phosphoinositide 3 kinase; cAMP- cyclic AMP; PKA- protein kinase cAMP-dependent; $\uparrow$ - stimulation; $\mathrm{T}$ - inhibition.

cellular remodeling have yet to be established [92]. Some factors that can trigger this event in vitro have been characterized, such as low $\mathrm{pH}$, lack of oxygen and nutritional depletion. Interestingly, endosome sorting and autophagy are required for metacyclogenesis [93,95-97].

The recent sequencing of the genome of three species of trypanosomatids has revealed the presence of several proteins involved in specific signaling pathways, such as intermediate messengers and kinases [82,98]. Leishmania major appears to have 179 protein kinases that are similar to eukaryotic counterparts, representing $2 \%$ of the Leishmania genome $[9,99]$. Nevertheless, the trypanosomatids lack receptor linked and cytosolic tyrosine kinase families and some groups of protein kinases, such as STE and GMGC (CDK cyclin-dependent kinases, MAP kinases, glycogen synthase kinase 3 kinase-dependent cell division cycle (CDC) as and casein kinase II family) are involved in important processes, including cell cycle regulation, stress responses, cellular signals and cell differentiation $[9,82]$. Moreover, some genes were found for certain families of protein kinases, such as CAMK $\left(\mathrm{Ca}^{+2}\right.$ regulated by kinases) and $\mathrm{AGC}$ (which includes the protein kinase $\mathrm{C}$, protein kinase $A$, protein kinase $G$ etc) [9].

The MAP kinase family of proteins is involved in the regulation of cellular proliferation and differentiation in eukaryotic cells $[9,100]$. Fifteen MAPK genes were identified in L. major and L. mexicana, which are also present in $L$. infantum and L. braziliensis $[99,47]$. Studies using L. major and $L$. donovani showed that the increase in the recombinant MPK 4, MPK 7 and MPK 10 activity was similar to that observed during differentiation to the amastigote form. Additionally, MPK 3, MPK 13 and MPK 9 seem to be involved in the elongation and reduction of the flagellar length in $L$. mexicana and may be implicated in the regulation of cell differentiation [82,99,101-104]. Earlier reports suggested that MPK 4 in L. mexicana may have an important role in the progression of promastigotes to amastigotes because the $T$. brucei homologue of the enzyme TbMAPK2 null mutant showed a delay in differentiation kinetics [80]. Although several studies have highlighted the importance of MAP kinase proteins in cell differentiation, the signaling pathways involved in its activation are still unknown. Unusual surface receptors, the kinase tyrosine receptor, conventional substrates and transcription factors $[99,47]$ are present in the Leishmania genome.

cAMP is an important second messenger that modulates several key cell processes. The Leishmania genome project showed that the parasite contains members of the cAMP cascade [35,105]. In Leishmania, studies have demonstrated that stress-induced elevations of the intracellular cAMP response are associated with cellular differentiation. Thus, cAMP may play a significant role in stress-induced differentiation because both intracellular cAMP and the activity of protein kinase A are elevated under stress [105].

Despite the fact that few members of the AGC kinase family have been identified in the trypanosomatid genome, three isoforms of PKA-C (catalytic subunit) and possibly an isoform of PKA-R (regulatory subunit) have been characterized in Leishmania. PKA is a tetrameric cAMP-dependent protein kinase that can modulate signaling pathways involved in cellular proliferation and differentiation processes $[47,106,107]$. However, unlike higher eukaryotes, very little is known about the role of PKA in Leishmania. In 2004, PKA activity was found to be higher in metacyclic forms compared to non-infective forms, which may suggest the 
involvement of the enzyme in the process of parasite metacyclogenesis [108].

The presentation of the Leishmania genome showed the occurrence of gene members of environmental signaling pathways that may have an important role in the cell differentiation of these parasites. However, the actual contribution of these pathways to this process has yet to be determined.

\section{PERSPECTIVES}

The study of signaling pathways in trypanosomes is crucial for understanding the relationship of these parasites with their hosts (both vertebrates and invertebrates). Even so, our knowledge in this field today is still limited. To date, most studies of intracellular signaling pathways in trypanosomatids have used the simple algorithm of a single input or ligand to address the role of a single pathway or a small number of signaling pathways (Fig. 3). This strategy was largely hampered by the unavailability of genomic and proteomic data. However, the first wide and more complex maps of cell signaling in such organisms could be constructed in the coming years. This undertaking will certainly be supported by both the availability of the data on parasite biology and bioinformatics and by the surge in novel high-throughput technologies to evaluate cell signaling and protein phosphorylationdephosphorylation circuits. The first products of this new phase are becoming available in the literature, as are data generated on kinome, phosphatome and phosphoproteome projects in some groups of pathogenic trypanosomatids [4547]. However, while such studies present an outstanding account of enzymes encoded in protozoan genomes and could eventually lead to the full collection of their target phosphoproteins, they are an initial platform for future investigation. It is of the utmost importance to put conventional signaling modulators of trypanosomatid biology on the map and to establish a detailed picture of the connection between molecules traditionally under study and their modes of action. We need to identify very specific signaling molecules or motifs within conventionally-studied molecules that could be a starting point for the development of novel targets to block diseases caused by trypanosomatids. These studies should take the place of those describing canonical signaling scaffolds in pathogenic protozoa. In conclusion, further investigation of the modulation of cellular differentiation in trypanosomatids with the aid of these novel technologies is of the utmost importance. Determining how the signaling pathways may contain potential targets for the development of immunotherapeutic and chemotherapeutic drugs in future years should be another research priority.

\section{ACKNOWLEDGEMENTS}

We dedicate this review to Jayme Angluster, who led our research for many years. We are grateful to Patricia Lourenço Dutra and Claudia Oliveira Rodrigues for their contributions to this work for more than a decade. The authors wish to express their gratitude to Mr. João Müller Haddad who kindly drew the cartoons depicted in this review. These studies were supported by the Brazilian financial agencies Conselho Nacional de Desenvolvimento Cientifico e Tecnologico (CNPq), Coordenação de Aperfeiçoamento de Pessoal de Nível Superior (CAPES) and
Fundação de Amparo a Pesquisa Carlos Chagas Filho (FAPERJ) and also by the funding program "Instituto Nacional de Ciência e Tecnologia em Entomologia Molecular". This review is in honor of Carlos Chagas, because of Chagas disease centennial anniversary.

\section{REFERENCES}

[1] Andrade PP, Almeida DF. Environmental factors determining structural transition in Herpetomonas samuelpessoai. J Parasitol 1980; 66: 250-4.

[2] Fenn K, Matthews KR. The cell biology of Trypanosoma brucei differentiation. Curr Opin Microbiol 2007; 10: 539-46.

[3] Hoare CA, Wallace FG. Developmental stages of trypanosomatid flagellates: a new terminology. Nature 1966; 212: 1385-6.

[4] Svobodová M, Zídková L, Cepicka I, et al. Sergeia podlipaevi gen. nov., sp. nov. (Trypanosomatidae, Kinetoplastida), a parasite of biting midges (Ceratopogonidae, Diptera). Int J Syst Evol Microbiol 2007; 57: 423-32.

[5] Barcinski MA, Costa-Moreira ME. Cellular response of protozoan parasites to host-derived cytokines. Parasitol Today 1994; 10: 3525.

[6] Parsons M, Ruben L. Pathways involved in environmental sensing in trypanosomatids. Parasitol Today 2000; 16: 56-62.

[7] El-Sayed NM, Myler PJ, Bartholomeu DC, et al. The genome sequence of Trypanosoma cruzi, etiologic agent of Chagas disease. Science. 2005; 309: 409-15.

[8] Berriman M, Ghedin E, Hertz-Fowler C, et al. The genome of the African trypanosome Trypanosoma brucei. Science 2005; 309: 416-22.

[9] Ivens AC, Peacock CS, Worthey EA, et al. The genome of the kinetoplastid parasite, Leishmania major. Science 2005; 309: 43642.

[10] Lopes AHCS, Costa e Silva Filho F, Alviano CS, et al. Changes in cell surface anionogenic groups induced by propranolol in Herpetomonas muscarum muscarum. J Protozool 1989; 36: 231-7.

[11] Dedet JP, Roche B, Pratlong F, et al. Diffuse cutaneous infection caused by a presumed monoxenous trypanosomatid in a patient infected with HIV. Trans R Soc Trop Med Hyg 1995; 89: 644-6.

[12] Boisseau-Gersaud AM, Cales-Quist D, Desbois N, et al. A new case of cutaneous infections by a presumed monoxenous trypanosomatid in the island of Martinique. Trans R Soc Trop Med Hyg 2000; 94: 51-2.

[13] Chao W, Olson M. Platelet-activating factor: receptors and signal transduction. Biochem J 1993; 292: 617-29.

[14] Lopes AH, Dutra PM, Rodrigues CO, et al. Effect of plateletactivating factor on the process of cellular differentiation of Herpetomonas muscarum muscarum. J Eukaryot Microbiol 1997; 44: 321-5.

[15] Rodrigues CO, Dutra PM, Souto-Padrón T, Cordeiro RS, Lopes AH. Effect of platelet-activating factor on cell differentiation of Trypanosoma cruzi. Biochem Biophys Res Commun 1996; 223: 735-40.

[16] Silva-Neto MA, Carneiro AB, Vieira DP, Mesquita RD, Lopes AH Platelet-activating factor (PAF) activates casein kinase 2 in the protozoan parasite Herpetomonas muscarum muscarum. Biochem Biophys Res Commun 2002; 293: 1358-63.

[17] Gomes MT, Monteiro RQ, Grillo LA, et al. Platelet-activating factor-like isolated from Trypanosoma cruzi. Int J Parasit 2006; 36: 165-73.

[18] Naula C, Parsons M, Mottram JC. Protein kinases as drug targets in trypanosomes and Leishmania. Biochem Biophys Acta 2005; 1754: 151-9.

[19] Stuart K, Brun R, Croft S, et al. Kinetoplastids: related protozoan pathogens, different diseases. J Clin Invest 2008; 118: 1301-10.

[20] Contreras VT, Morel CM, Goldenberg S. Stage specific gene expression precedes morphological changes during Trypanosoma cruzi metacyclogenesis. Mol Biochem Parasitol 1985; 14: 83-96.

[21] Adade CM, Cons BL, Melo PA, Souto-Padrón T. Effect of Crotalus viridis viridis snake venom on the ultrastructure and intracellular survival of Trypanosoma cruzi. Parasitology 2010; 21: 1-13.

[22] Fraidenraich D, Peña C, Isola EL, et al. Stimulation of Trypanosoma cruzi adenyl-cyclase by an LD-globin fragment from Triatoma hindgut. Effect on differentiation of epimastigote to trypomastigote forms. Proc Natl Acad Sci USA 1993; 90: 10140-4. 
[23] Ouaissi MA, Cornette J, Schoneck R, et al. Fibronectin cleavage fragments provide a growth factor-like activity for the differentiation of Trypanosoma cruzi trypomastigotes to amastigotes. Eur $\mathbf{J}$ Cell Biol 1992; 59: 68-79.

[24] Castro SL, Meirelles MNL, Oliveira MM. Trypanosoma cruzi: adrenergic modulation of cyclic AMP role in proliferation and differentiation of amastigotes in vitro. Exp Parasitol 1988; 64: 368-75.

[25] Gonzales-Perdomo M, Romero P, Goldenberg S. Cyclic AMP and adenylate cyclase activators stimulate Trypanososma cruzi differentiation. Exp Parasitol 1988; 66: 205-12.

[26] Oliveira MM, Antunes A, Mello FG. Growth of Tryponosoma cruzi epimastigotes controlled by shifts in AMP mediated by adrenergic ligands. Mol Biochem Parasitol 1984; 11: 283-92.

[27] Oliveira MM, Rocha ED, Rondinelli E, Arnholdt AV, Scharfstein J. Signal transduction in Trypanosoma cruzi: opposite effects of adenylcyclase and phospholipase $\mathrm{C}$ systems in growth control. Mol Cell Biochem 1993; 124: 91-9.

[28] Laxman S, Beavo JA. Cyclic nucleotide signaling mechanisms in trypanosomes: possible targets for terapeutics agents. Mol Interv 2007; 7: 203-15.

[29] Shalaby T, Liniger M, Seebeck T. The regulatory subunit of a cGMP-regulated protein kinase A of Trypanosoma brucei. Eur J Biochem 2001; 268: 6197-206.

[30] Ulloa RM, Mesri E, Esteva M, Torres HN, Tellez-Inon MT. Cyclic AMP-dependent protein kinase activity in Trypanosoma cruzi. Biochem J 1988; 255: 319-26.

[31] Huang H, Werner C, Weiss LM, Wittner M, Orr GA. Molecular cloning and expression of the catalytic subunit of protein kinase A from Trypanosoma cruzi. Int J Parasitol 2002; 32: 1107-15.

[32] Banerjee C, Sarkar D. Isolation and characterization of a cyclic nucleotide-independent protein kinase from Leishmania donovani. Mol Biochem Parasitol 1992; 52: 195-205.

[33] Siman-Tov MM, Aly R, Shapira M, Jaffe CL. Cloning from Leishmania major of a developmentally regulated gene, c-lpk2, for the catalytic subunit of the cAMP-dependent protein kinase. Mol Biochem Parasitol 1966; 77: 201-15.

[34] Bao Y, Weiss LM, Braunstein VL, Huang H. Role of Protein Kinase A in Trypanosoma cruzi. Infect Immun 2008; 4757-63.

[35] Naula C, Seebeck T. Cyclic AMP Signaling in Trypanosomatids. Parasitol Today 2000; 16: 35-8.

[36] Taylor MC, Muhia DK, Baker DA, et al. Trypanosoma cruzi adenylyl cyclase is encoded by a complex multigene family. Mol Biochem Parasitol 1999; 104: 205-17.

[37] Coso OA, Díaz-Añel A, Martinetto H, et al. Characterization of a Gi-protein from Trypanosoma cruzi epimastigote membranes. Biochem J 1992; 287: 443-336.

[38] D'Angelo MA, Montagna AE, Sanguineti S, Torres HN, Flawiá MM. A novel calcium-stimulated adenylyl cyclase from Trypanosoma cruzi, which interacts with the structural flagellar protein paraflagellar rod. J Biol Chem 2002; 277: 35025-34.

[39] Garrido M, Bollo M, Machado-Domenech E. Biphasic and dosedependent accumulation of INSP3 in Trypanosoma cruzi stimulated by a synthetic peptide carrying a chicken $\alpha^{\mathrm{D}}$-globin fragment. Cell Mol Biol 1996; 42: 850-64.

[40] Santander V, Bollo M, Machado-Domenech E. Lipid kinases and $\mathrm{Ca}^{+2}$ signaling in Trypanosoma cruzi stimulated by a synthethic peptide. Biochem Biophys Res Commun 2002; 293: 314-20.

[41] Wainszelbaum MJ, Belaunzarán ML, Lammel EM, et al. Free fatty acids induce cell differentiation to infective forms in Trypanosoma cruzi. Biochem J 2003; 375: 705-12.

[42] Belaunzarán ML, Lammel EM, Giménez G, Wainszelbaum MJ, Isola ELD. Involvement of protein kinase C isoenzymes in Trypanosoma cruzi metacyclogenesis induced by oleic acid. Parasitol Res 2009; 105: 47-55.

[43] Avila AR, Dallagiovanna B, Yamada-Ogatta SF, et al. Stagespecific gene expression during Trypanosoma cruzi metaciclogenesis. Genet Mol Res 2003; 2: 159-68.

[44] Rodrigues CO, Dutra PML, Barros FS, et al. Platelet-activating factor induction of secreted phosphatase activity in Trypanosoma cruzi. Biochem Biophys Res Commun 1999; 266: 36-42.

[45] Brenchley R, Tariq H, McElhinney H, et al. The TriTryp phosphatome: analysis of the protein phosphatase catalytic domains. BMC Genomics 2007; 8: 434-55.

[46] Nakayasu ES, Gaynor MR, Sobreira TJP, Ross JA, Almeida IC. Phosphoproteomic analysis of the human pathogen Trypanosoma cruzi at the epimastigote stage. Proteomics 2009; 9: 3489-506.
[47] Parsons M, Worthey EA, Ward PN, Mottram JC. Comparative analysis of the kinomes of three pathogenic trypanosomatids: Leishmania major, Trypanosoma brucei and Trypanosoma cruzi. BMC Genomics 2005; 6: 127-46.

[48] Reuner B, Vassella E, Yutzy B, Boshart M. Cell density triggers slender to stumpy differentiation of Trypanosoma brucei bloodstream forms in culture. Mol Biochem Parasitol 1997; 90: 269-80.

[49] Vassella E, Reuner B, Yutzy B, Boshart M. Differentiation of African trypanosomes is controlled by a density sensing mechanism which signals cell cycle arrest via the cAMP pathway. J Cell Sci 1997; 110: 2661-71.

[50] Welburn SC, Maudlin I. Tsetse-trypanosome interactions: rites of passage. Parasitol Today 1999; 15: 399-403.

[51] Cross GAM. Identification, purification and properties of clonespecific glycoprotein antigens constituting the surface coat of Trypanosoma brucei. Parasitology 1975; 71: 393-417.

[52] Roditi I, Clayton C. An unambiguous nomenclature for the major surface glycoproteins of the procyclic form of Trypanosoma brucei. Mol Biochem Parasitology 1999; 103: 99-100.

[53] Ferguson MAJ. The structure, biosynthesis and functions of glycosylphosphatidylinositol anchors, and the contributions of trypanosome research. J Cell Sci 1999; 112: 2799-809.

[54] Overath P, Czichos J, Stock U, Nonnengaesser C. Repression of glycoprotein synthesis and release of surface coat during differentiation of Trypanosoma brucei. EMBO J 1983; 2: 1721-8.

[55] Roditi I, Schwarz H, Pearson TW, et al. Procyclin gene expression and loss of the variant surface glycoprotein during differentiation of Trypanosoma brucei. J Cell Biol 1989; 108: 737-46.

[56] Gruszynski AE, van Deursen F J, Albareda M C, et al. Regulation of surface coat exchange by differentiating African trypanosomes. Mol Biochem Parasitol 2006; 147: 211-23.

[57] Bulow R, Nonnengasser C, Overath P. Release of the variant glycoprotein during differentiation of bloodstream to procyclic forms of Trypanosoma brucei. Mol Biochem Parasitol 1989; 32: 85-92.

[58] Ziegelbauer K, Stahl B, Karas M, Stierhof Y-D, Overath P. Proteolytic release of cell surface proteins during differentiation of Trypanosoma brucei. Biochemistry 1993; 32: 3737-42.

[59] Gruszynski AE, DeMaster A, Hooper NM, Bangs JD. Surface coat remodeling during differentiation of Trypanosoma brucei. J Biol Chem 2003; 278: 24665-72.

[60] Carrington MR, Bulow R, Reinke H, Overath P. Sequence and expression of the glycosyl-phosphatidylinositol-specific phospholipase C of Trypanosoma brucei. Mol Biochem Parasitol 1989; 33: 289-96.

[61] Hereld D, Krakow JL, Bangs JD, Hart GW, Englund PT. A phospholipase C from Trypanosoma brucei which selectively cleaves the glycolipid on the variant surface glycoprotein. J Biol Chem 1986; 261: 13813-9.

[62] El-Sayed NMA, Donelson JE. African trypanosomes have differentially expressed genes encoding homologues of Leishmania GP63 surface protease. J Biol Chem 1997; 272: 26742-8.

[63] LaCount DJ, Gruszynski AE, Grandgenett PM, Bangs JD, Donelson JE. Expression and function of the Trypanosoma brucei major surface protease (GP63) genes. J Biol Chem 2003; 278: 24658-64.

[64] Krakow JL, Hereld D, Bangs JD, Hart GW, Englund PT. Identification of a glycolipid precursor of the Trypanosoma brucei variant surface glycoprotein. J Biol Chem 1986; 261:12147-53.

[65] Menon AK, Mayor S, Ferguson MAJ, Duszenko M, Cross GAM. Candidate glycophospholipid precursor of the glycosylphosphatidylinositol membrane anchor of Trypanosoma brucei variant surface glycoprotein. J Biol Chem 1988; 263: 1970-77.

[66] Field MC, Menon AK, Cross GAM. Developmental variation of glycosylphosphatidylinositol membrane anchors in Trypanosoma brucei. Identification of a candidate biosynthetic precursor of the glycosylphosphatidylinositol anchor of the major procyclic stage surface glycoprotein. J Biol Chem 1991; 266: 8392-400.

[67] Brun R, Schonenberger M. Stimulating effect of citrate and cisaconitate on the transformation of Trypanosoma brucei bloodstream forms to procyclic forms in vitro. Z Parasitenkd 1981; 66: $17-24$.

[68] Czichos J, Nonnengaesser C, Overath P. Trypanosoma brucei: cisaconitate and temperature reduction as triggers of synchronous transformation of bloodstream to procyclic trypomastigotes in vitro. Exp Parasitol 1986; 62: 283-91. 
[69] Overath P, Czichos J, Haas C. The effect of citrate:cisaconitate on oxidative metabolism during transformation of Trypanosoma brucei. Eur J Biochem 1986; 160: 175-82.

[70] Engstler M, Boshart M. Cold shock and regulation of surface protein trafficking convey sensitization to inducers of stage differentiation in Trypanosoma brucei. Genes Dev 2004; 18: 2798-811.

[71] Rotureau B, Morales MA, Bastin P, Spath GF. The flagellummitogen-activated protein kinase connection in trypanosomatids: a key sensory role in parasite signaling and development? Cell Microbiol 2009; 11: 710-18.

[72] Nett IRE, Martin DMA, Miranda-Saavedra D, et al. The phosphoproteome of bloodstream form Trypanosoma brucei, causative agent of African sleeping sickness. Mol Cell Proteomics 2009; 8: 1527-38.

[73] Parsons M, Valentine M, Deans J, Schieven GL, Ledbetter JA. Distinct patterns of tyrosine phosphorylation during the life cycle of Trypanosoma brucei. Mol Biochem Parasitol 1991; 45: 241-8.

[74] Cool DE, Blum JJ. Protein tyrosine phosphatase activity in Leishmania donovani. Mol Cell Biochem 1993; 127-128: 143-9.

[75] Bakalara N, Seyfang A, Baltz T, Davis C. Trypanosoma brucei and Trypanosoma cruzi: life cycle-regulated protein tyrosine phosphatase activity. Exp Parasitol 1995; 81: 302-12.

[76] Szöor B, Wilson J, McElhinney H, Tabernero L, Matthews KR. Protein tyrosine phosphatase TbPTP1: a molecular switch controlling life cycle differentiation in trypanosomes. J Cell Biol 2006; 23: 293-303.

[77] Shapiro SZ, Naessens J, Liesegang B, Moloo SK, Magondu J. Analysis by flow cytometry of DNA synthesis during the life cycle of African trypanosomes. Acta Trop 1984; 41: 313-23.

[78] Wiesmann C, Barr KJ, Kung J, et al. Allosteric inhibition of protein tyrosine phosphatase 1B. Nat Struct Mol Biol 2004; 11: 730-7.

[79] Hua SB, Wang CC. Differential accumulation of a protein kinase homolog in Trypanosoma brucei. J Cell Biochem 1994; 54: 20-31.

[80] Muller IB, Domenicali-Pfister D, Roditi I, Vassella E. Stage specific requirement of a mitogen-activated protein kinase by Trypanosoma brucei. Mol Biol Cell 2002; 13: 3787-99.

[81] Domenical-Pfister D, Burkard G, Morand S, et al. Mitogenactivated protein kinase controls differentiation of bloodstream forms of Trypanosoma brucei. Eukaryot Cell 2006; 5: 1126-35.

[82] Rotureau B, Morales MA, Bastin P, Spath GF. The flagellummitogen-activated protein kinase connection in trypanosomatids: a key sensory role in parasite signaling and development? Cell Microbiol 2009; 11: 710-8.

[83] Strickler JE, Patton CL. Adenosine 3',5'-monophosphate in reproducing and differentiated trypanosomes. Science 1975; 190: 11102 .

[84] Mancini PE, Patton CL. Cyclic 3',5'-adenosine monophosphate levels during the developmental cycle of Trypanosoma brucei bruce $i$ in the rat. Mol Biochem Parasitol 1981; 3: 19-31.

[85] Laxman S, Riechers A, Sadilek M, Schwede F, Beavo JA. Hydrolysis products of cAMP analogs cause transformation of Trypanosoma brucei from slender to stumpy-like forms. PNAS 2006; 50: 19194-9.

[86] Dean S, Marchetti R, Kirk K, Matthews KR. A surface transporter family conveys the trypanosome differentiation signal. Nature 2009; 459: 213-7.

[87] Santos CC, Coombs GH, Lima APCA, Mottram JC. Role of the Trypanosoma brucei natural cysteine peptidase inhibitor ICP in differentiaton and virulence. Mol Microbiol 2007; 66: 991-1002.

[88] Hendriks EF, Matthews KR. Disruption of the developmental programme of Trypanosoma brucei by genetic ablation of TbZFP1, a differentiation-enriched CCCH protein. Mol Microbiol 2005; 57: 706-16.

[89] Stodjl DF, Clarke MW. Trypanosoma brucei: analysis of cytoplasmic $\mathrm{Ca}^{2+}$ during differentiation of bloodstream stages in vitro. Exp Parasitol 1996; 83: 134-46.

[90] Desjeux P. Leishmaniasis. Public health aspects and control. Clin Dermatol 1996; 14: 417-23.

[91] Cohen-Freue G, Holzer TR, Forney JD, McMaster WR. Global gene expression in Leishmania. Int J Parasitol 2007; 37: 1077-86.

[92] Bates, PA, Rogers, ME. New insights into the developmental biology and transmission mechanisms of Leishmania. Curr Mol Med 2004; 4: 601-9.

[93] Besteiro S, Williams RAM, Coombs GH, Mottram JC. Protein turnover and differentiation in Leishmania. Int J Parasitol 2007; 37 : 1063-75.

[94] Pimenta PFP, Turco SJ, McConville MJ, et al. Stage-specific adhesion of Leishmania promastigotes to the sandfly midgut. Science 1992; 256: 1812-5.

[95] Besteiro S, Williams RAM, Morrison LS, Coombs GH, Mottram JC. Endodome sorting and autophagy are essential for differentiation and virulence of Leishmania major. J Biol Chem 2006; 281: 11384-96.

[96] Bates PA. Leishmania sandfly interaction: progress and challenges Cur Opin Microbiol 2008; 11: 340-4.

[97] Barak E, Amin-Spector S, Gerliak E, et al. Differentiation of Leishmania donovani in host-free system: analysis of signal perception and response. Mol Biochem Parasitol 2005; 141: 99-108.

[98] El-Sayed NM, Myler PJ, Blandin G, et al. Comparative genomics of trypanosomatid parasitic protozoa. Science 2005; 309: 404-9.

[99] Wiese M. Leishmania MAP kinases - familiar proteins in an unusual context. Int J Parasitol 2007; 37: 1053-62.

[100] Sebolt-Leopold JS, Herrera R. Targeting the mitogen-activated protein kinase cascade to treat cancer. Nat Rev Cancer 2004; 4: 937-47.

[101] Kuhn D, Wiese M. LmxPK4, a mitogen-activated protein kinase kinase homologue of Leishmania mexicana with a potential role in parasite differentiation. Mol Microbiol 2005; 56: 1169-82.

[102] Bengs F, Scholz A, Kuhn D, Wiese M. LmxMPK9, a mitogen activated protein kinase homologue affects flagellar length in Leishmania mexicana. Mol Microbiol 2005; 55: 1606-15.

[103] Erdmann M, Scholz A, Melzer IM, Schmetz C, Wiese M. Interacting protein kinases involved in the regulation of flagellar length. Mol Biol Cell 2006; 17: 2035-45.

[104] Morales MA, Renaud O, Faigle W, Shorte SL, Spath GF. Overexpression of Leishmania major MAP kinases reveals stagespecific induction of phosphotransferase activity. Int J Parasitol 2007; 37: 1187-99.

[105] Bhattacharya A, Biswas A, Das PK. Role of intracellular cAMP in differentiation-coupled induction of resistance against oxidative damage in Leishmania donovani. Free Rad Biol Med 2008; 44: 779-94.

[106] Dell KR, Engel JN. Stage-specific regulation of protein phosphorylation in Leishmania major. Mol Biochem Parasitol 1994; 64: 28392.

[107] Malki-Feldman L, Jaffe CL. Leishmania major: effect of protein kinase $\mathrm{A}$ and phosphodiesterase activity on infectivity and proliferation of promastigotes. Exp Parasitol 2009; 123: 39-44.

[108] Genestra M, Cysne-Finkelstein L, Leon L. Protein kinase A activity is associated with metacyclogenesis in Leishmania amazonensis. Cell Biochem Funct 2004; 22: 315-20. 